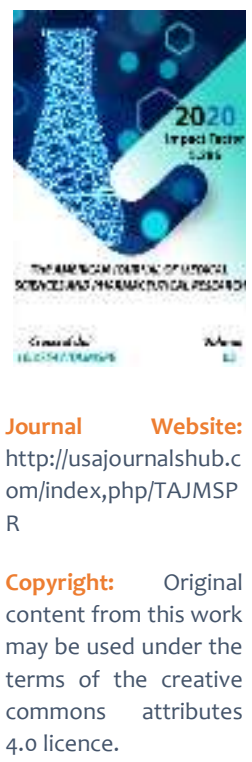

\title{
Glial Tumors Of The Brain, With Associated Hydrocephalia
}

\author{
Ruzikulov M.M. \\ Tashkent Pediatric Medical Institute, Uzbekistan \\ Mukhammedaminov B.Sh. \\ Tashkent Pediatric Medical Institute, Uzbekistan \\ Rahimov I.I. \\ Tashkent Pediatric Medical Institute, Uzbekistan
}

Burnashev M.I.

Republican Specialized Scientific Practical Medical Center Of Neurosurgery Of Uzbekistan

Kim A.A.

Republican Specialized Scientific Practical Medical Center Of Neurosurgery Of Uzbekistan

\section{ABSTRACT}

Despite significant advances in oncology and neurosurgery, glial brain tumors continue to be an urgent problem of modern healthcare. The proportion of gliomas in the total structure of glial neoplasms is $40-45 \%$, mainly they are detected at the age of 30-60 years, affecting the most able-bodied part of the population. Glial neoplasms, as a rule, develop from cells of the astrocytic or oligodendrocytic population and are characterized by a rapid growth rate of the primary tumor node, invasiveness, a tendency to early metastasis, a high recurrence rate, and a poor prognosis. The primary importance of tumor removal in the treatment of the disease is also generally recognized in cases where the pathological process is complicated by persistent disorders of the cerebrospinal fluid, deformation and expansion of the cerebrospinal fluid cavities. Naturally, the development of hydrocephalus, especially hydrocephalic-hypertensive syndrome, changes the course and prognosis of the disease and requires significant correction of the diagnostic and therapeutic process. As with most malignant tumors, anaplastic types of gliomas are characterized by the intensive development of the pathological vasculature, which accelerates the growth rate of the neoplasm, the rate of invasion and metastasis, and also increases the risk for patients due to the possibility of hemorrhage into the tumor. The nodular type of growth with a clearly defined border and insignificant infiltration is less common in conditionally benign gliomas, which have a more favorable prognosis of treatment. In the end, it is concluded that the described methods do not exhaust all proposals for increasing the effectiveness of the treatment of glial tumors and the development of new methods will bring neurooncologists closer to solving this urgent problem.

\section{KEYWORDS}

intracranial tumors, hydrocephalus, intracranial hypertension. 


\section{INTRODUCTION}

Tumor to the central nervous system occurs in 15-30 cases per 1 million of the population annually. Over the past 20 years, the incidence of brain tumors has increased by $2.7 \%$ per year $[1,6,12,17]$. Hydrocephalus occurs in $20-96 \%$ of patients, depending on the location, histostructure and size of the brain neoplasm and the patient's age $[3,4,9,10,13,23]$. The primary importance of tumor removal in the treatment of the disease is also generally recognized in cases where the pathological process is complicated by persistent disorders of the cerebrospinal fluid, deformation and expansion of the cerebrospinal fluid cavities. Naturally, the development of hydrocephalus, especially hydrocephalic-hypertensive syndrome, changes the course and prognosis of the disease and requires significant correction of the diagnostic and therapeutic process. In questions concerning the solution of specific aspects of the pathogenesis, diagnosis and treatment of patients with brain tumors complicated by hydrocephalus, however, there is no unanimity, and many questions are far from being solved.

\section{MATERIAL AND METHODS}

A retrospective analysis of the results of examination and surgical treatment of 414 children with brain tumors at the age of 3 months and more was carried out. up to 17 years for the period from 1996 to 2006 The age of the majority of patients ranged from 4 to 12 years (70.3\%). To determine the nature of tumor growth, to study the features of deformation of liquor-containing cavities and their severity, to determine the nature and severity of disorders of CSF circulation, the results of clinical and CSF dynamic studies were compared. The localization, type of growth, size and histostructure of the tumor, as well as the degree of anaplasia (I, II, III, IV grade) and metastasis ( $\mathrm{M} \mathrm{O}, \mathrm{I}, \mathrm{II}, \mathrm{III}, \mathrm{IV}$ ) of the blastomatous process were determined. For this purpose, the results of neurological, neuroophthalmological, otoneurological, ultrasound studies (US), computed tomography (CT), magnetic resonance imaging (MRI), positron emission tomography (PET), as well as intraoperative ultrasound monitoring and morphometry, histological analysis of biopsy material. In some cases, control of the degree of removal of the neoplasm was carried out intraoperatively using endoscopic techniques. A quantitative assessment of the severity of hydrocephalus was carried out by determining the cephaloventricular coefficients, production rate, resistance to resorption of cerebrospinal fluid, using ultrasound, CT, MRI and CSF dynamic studies.

As a result of the research and treatment, a data bank was created, taking into account 116 signs. Statistical analysis was carried out on the basis of the available tables of values from the general population by compiling contingency tables and constructing sample histograms for an initial assessment of the distribution of features. For each feature, the observed and expected sample probabilities were determined, and the null hypothesis was tested using the $\chi^{2}$ test. The statistical significance of the differences was assessed. The difference was considered significant at $p$ $<0.05$. At $p<0.1 \mathrm{p}<0.3$, a trend was observed.

\section{SURVEY RESULTS}

The study group consisted of children with cerebral neoplasms of III and IV degrees of anaplasia - 216 (52.2\%). The control group included patients with grade I-II OGM. anaplasias - 198 (47.8\%). As expected, patients 
with intracerebral neoplasms prevailed - 320 (77.3\%). There were 265 tumors of the posterior cranial fossa (64\%), supratentorial neoplasms 149 (36\%). Only $9.8 \%$ of patients had tumor volume less than $30 \mathrm{cc}$. At the same time, in $48.2 \%$ of observations, large (more than 60 cubic $\mathrm{cm}$ ) neoplasms were revealed. There were no significant differences between tumor sizes in the groups of patients with benign and malignant neoplasms. Despite the fact that malignant tumors were 1.2 times more likely to have subtentorial localization, this difference was not statistically significant ( $p>0.5$ ). Hydrocephalus of varying severity was found in 331 (79.9\%) patients. When assessing the severity of dropsy of the brain in both groups, its reliable relationship with the degree of anaplasia of the neoplasm was not revealed (Fig. 1). Analysis of the relationship between the localization of the tumor and the presence of hydrocephalus shows that severe hydrocephalus was more often detected with subtentorial localization of the blastomatous process, and normoventriculi microventriculum was more typical for supratentorial neoplasms ( $p<0.05)$ (Table 1$)$. The relationship between the child's age and the presence of hydrocephalus was revealed. Dropsy of the brain of varying severity prevailed significantly in infancy - 26 (83.8\%) out of 31 and at preschool age 153 (71.2\%) out of 215. Features of manifestations of hydrocephalus in malignant brain tumors. In malignant brain tumors, hydrocephalic syndrome of varying severity was detected in $134(62 \%)$ patients out of 216 . At the same time, severe dropsy occurred in 34 (25.3\%) cases. Severe or moderately severe hydrocephalus was equally often detected in the blastomatous process of III and IV degrees of anaplasia. Patients with malignant brain tumors, combined with severe hydrocephalus, were assessed as severe or extremely severe in
78.7\% of cases, and with mildly severe hydrocephalus or absence of dropsy, it was observed in only $31.5 \%(\mathrm{p}<0.01)$ patients. In the presence of severe hydrocephalus in patients with malignant brain tumors, the course of the pathological process was rapid or progressive in $92.6 \%$ of cases, and in its absence it was noted in $66.2 \%$ ( $p<0.03$ ). The frequency of detection of hydrocephalus depended on the location, size, type of tumor growth. As expected, severe hydrocephalus was noted 5.1 times more often in medium and large tumors than in small neoplasms ( $p$ <0.01). Hydrocephalus was more often observed when the tumor was localized in the posterior cranial fossa, third ventricle, and median neoplasms. In diffuse midline tumors, hydrocephalus was established more often than in nodular hemispheric neoplasms ( $p$ $<0.05)$. Occlusive dropsy was detected in 166 (76.8\%) patients, and communicating - in 70 (32.4\%) ( $p<0.05)$. At the same time, severe hydrocephalus was assessed as occlusive in 133 (78.3\%) cases out of 166 , and communicating in $42(60 \%)$ out of 70 . In malignant tumors, signs of occlusion of the cerebrospinal fluid were revealed 1.6 times less often and 1.8 Signs of CSF hyperproduction more often than in benign brain tumors ( $p<0.03$ and $p<0.05$, respectively). It was important that in persons with malignant types of blastomatous growth, pronounced peritumorous edema was detected 2.7 times more often, than with benign neoplasms ( $p<0.01)$. In the presence of tumor metastases (M1-M4), hydrocephalus was assessed as reported 1.9 times more often than in their absence ( $p<0.05)$. Thus, the development of cerebrospinal fluid circulation disorders and a significant expansion of the cerebrospinal fluid cavities are accompanied by a deterioration in the condition of neurooncological patients and aggravates the course of the pathological process. 


\section{TREATMENT RESULTS}

In both groups, total and subtotal tumor resection was performed in 173 (41.8\%) 187 ( $45.2 \%)$ cases, respectively. At the same time, in the study group, complete removal of the neoplasm was achieved 1.9 times less often than in the control group ( $p<0.01)$. Regression of hydrocephalus was approximately equally achieved after total and subtotal neoplasm resection ( $71.6 \%$ and $66.2 \%$, respectively). It should be noted that after partial removal of Brain tumors dropsy remained in $43.3 \%$. The dynamics of the hydrocephalic syndrome of removal depended on the size of the tumor, localization and type of growth. Seven patients ( 5 from the study group and 2 from the control group) underwent cerebrospinal fluid shunting (ventriculoperitoneostomy in 6 patients and ventriculoatriostomy in 1) before removal of the neoplasm in order to stabilize the condition of patients with hydrocephalic-hypertensivedislocation syndrome. In 5 cases, endoscopic perforation of the bottom of the 3 rd ventricle was performed before tumor removal. In 57 (13.7\%) cases, the removal of the tumor was completed by ventriculostomy, in which a message was formed between the ventricular system and the subarachnoid space, 51 (12.3\%) cases, after partial resection of the tumor or posterior parts of the third ventricle, performed ventriculocisternostomy according to Thorkildsen. In 4 cases, during the removal of the tumor, it became obvious that an Ventriculo-peritoneal shunting surgery should be performed and the operation ended with ventriculoperitoneostomy. After ventriculostomy, hydrocephalus control was achieved in $61.4 \%$ of cases, and ventriculocisternal anastomosis was effective in treating hydrocephalus in $72.5 \%$ of cases. Eliminating surgery was used to treat 68 patients with benign - $36(52.9 \%)$ and malignant brain tumors -32 (47.1\%), when removal of the neoplasm did not allow achieving control over the hydrocephalic-hypertensive syndrome, drug correction remained ineffective. In 43 (63.2\%) cases, before the implantation of the cerebrospinal fluid system, infusion-load tests were performed in patients after total or close to complete removal of the BM. In the overwhelming number of observations, violations of CSF resorption of one or another severity were revealed, in $10 \%$ hyperproduction of cerebrospinal fluid, in $23.4 \%$ of cases a combination of both mechanisms. After Ventriculo-peritoneal shunting, regression of hydrocephalus was achieved in $88.4 \%$ of patients. At the same time, the effectiveness of these operations in persons with benign and malignant brain tumors did not differ significantly. Complications of liquor shunt operations performed in the treatment of patients with malignant brain tumors complicated by the development of hydrocephalus were observed in $9(28.1 \%)$ cases. Shunt occlusion was most often noted $-27.5 \%$. It should be noted that this subgroup included all 4 observations with the simultaneous removal of tumor and ventriculoperitoneal shunting. In half of cases, shunt blockade was diagnosed within the first 6 months. after implantation of drainage, hypodrainage hyperdrainage states were noted in $27.5 \%$ and $13.3 \%$ of cases, respectively. Infectious complications were observed in 4.4\% of patients, and pseudoperitoneal cysts - in 5.1\%. Tumor metastasis through the shunt at the initial stages of the study was often observed in patients with malignant brain tumors (2.8\%), however, the use of V.P. Bersnev et al. shunts with an antimetastatic barrier containing a filter adsorbent, the occurrence of these complications was not observed in any of the 9 patients. Other complications were of a transient nature and responded to symptomatic treatment. It 
should be noted that 5 (55.5\%) patients had a combination of 2-4 different complications. In 3 cases, the relapse of the disease manifested itself at the initial stage with the progression of hydrocephalus; during introscopic examination of the patient in dynamics, a relapse of the tumor or secondary metastatic nodes along the craniospinal system were found.

\section{DISCUSSION OF RESULTS AND CONCLUSION}

In hydrocephalus and brain tumor are important and often mutually combining factors that determine the development of hypertensive-dislocation syndrome. The combination of these two mechanisms makes the course of malignant blastomatous growth especially rapid, in many respects complicating the prognosis of the disease. Our studies have shown that in patients with malignant brain tumors, severe or moderately severe dropsy is detected in $62 \%$ of patients. At the same time, with malignant neoplasms, dropsy is occlusive somewhat less often than with benign ones. The frequency, type and severity of hydrocephalus, in this case, depended on the nature of the blastomatous process and the age of the child. Dropsy of the brain is more often observed in median localized, medium large neoplasms, in children of younger age groups. Occlusive dropsy is more common with medianly localized tumors, and communicating - with laterally located ones. In malignant neoplasms, overproduction of CSF and a violation of its resorption are more often than in benign ones. While in benign tumors, on the contrary, the occlusive mechanism of the development of dropsy predominates, as evidenced by the literature $[2,3,4,8,9]$. In malignant neoplasms, the main mechanisms leading to the development of hypertensive or hypertensive-dislocation syndromes are the presence of an expansive process, excessive accumulation of CSF in the intracranial spaces and peritumorous edema. According to our data, pronounced peritumorous edema is detected in $2 / 3$ of patients with malignant brain tumors. Thus, these patients have not only dysregulation of the cerebrospinal fluid circulation, but also the accumulation of fluid in the vicinity of the cellular and interstitial spaces as a result of a violation of the waterelectrolyte balance. Then there is reason to assert that the Raimondi \& Mori model is adequate to characterize hydrocephalus in malignant blastomatous process, highlighting pericellular, interstitial, perocerebral and intraventricular CSF accumulation. Other mechanisms of the development of hypertensive hydrocephalus in malignant tumors, apparently, are due to intoxication caused by dysfunction of energy-dependent ion-transporting processes. The vulnerability of the cerebrospinal fluid regulation system, the rapid growth of neoplasms, a sharp deterioration in the condition of patients and a worsening prognosis of the disease make the problem of timely diagnosis of hydrocephalus in patients with malignant neoplasms of the brain a priority and their inclusion in the diagnostic complex is mandatory.

After removal of the tumor, hydrocephalus regresses in $67.2 \%$ of cases. This is especially clear when it comes to occlusive forms of hydrocephalus and tumor removal ensures the restoration of CSF outflow through the cerebrospinal fluid pathways. These data are in good agreement with the literature.

A brain tumor is an etiological factor responsible for the development of hydrocephalus. Therefore, removal of the tumor should naturally be attributed to etiotropic methods of treatment, even when the dropsy is not closed. After tumor removal, patients with malignant neoplasms have a high risk of recurrence or neoplasm procedure, i.e. 
re-emergence of the causes of hydrocephalus. Regarding the pathogenesis of hydrocephalus, in this case, the main mechanism can be considered a violation of CSF resorption due to a high level of protein and free tumor cells in the CSF, as well as infiltration of basal cisterns [17]. In those cases when, by removing the tumor, it was not possible to restore the ventriculo-subarachnoid cerebrospinal fluid, a ventriculo-subarachnoid anastomosis or ventriculostomy was performed. The use of these pathogenetic methods made it possible to achieve regression of hydrocephalus in $2 / 3$ of patients. These data are in good agreement with the literature obtained in the study of other similar objects $[3,4,9,11,14,18,23,24$, 25]. The use of cerebrospinal fluid shunting operations makes it possible to stabilize the state of patients with brain tumors complicated by the development of hydrocephalus, when the removal of the neoplasm, ventriculo-subarachnoid fistulas turned out to be ineffective. These operations are effective in all cases: when there is an occlusion of the cerebrospinal fluid, hyporesorption of the CSF or hypersecretion. Other authors also point out the universality of these operations. After these operations, however, the condition of patients in most cases becomes stable drainage-dependent. After CSF shunting operations, some serious complications developed on our material in $28.1 \%$ of cases. The high incidence of complications after cerebrospinal fluid shunting operations in patients with hydrocephalus, in general, and tumor genesis, in particular, were noted by other researchers $[3,4,5,8,9,10,14,15,16,17,19,22,23] \ldots$ In malignant neoplasms, a "specific" complication is observed - tumor metastasis through the shunt, which in our material was found in $2.8 \%$ of cases.
The use of special antimetastatic barriers can reduce the incidence of these formidable complications, but their risk still remains. Therefore, despite the high efficiency of these universal methods for correcting CSF circulation, their use is justified as symptomatic, when the use of etiotropic and pathogenetic methods is impossible or proved to be ineffective. The use of cerebrospinal fluid shunting operations before the removal of malignant tumors or simultaneously with its resections, although it allows to stabilize the patient's condition, however, in view of the high risk of unjustified surgical aggression and low efficiency compared to shunting operations after tumor removal, are advisable only in the presence of additional indications $[3,4,8,9,14,15,18,22]$. Based on our material, the course of the disease and the quality of life in patients with malignant brain tumors after removal of the neoplasm worsens while maintaining hydrocephalus, significantly improves after the corresponding hydrocephalus. Therefore, adequate correction of hydrocephalus should be an obligatory component of the complex treatment of malignant brain tumors. When choosing a treatment tactics, one should take into account both the patient's condition, the peculiarities of impaired circulation and deformation of the cerebrospinal fluid cavities, and the expected effectiveness of treatment and the prognosis of possible complications. At the same time, other things being equal, it is advisable to give preference to etiotropic, and then pathogenetic methods of treatment. The use of ventriculo-peritoneal shunting is justified when the use of the above methods was, for one reason or another, ineffective, impossible, or inappropriate.

During the observation period for patients with malignant brain tumors, the prognosis of treatment depends both on the characteristics 
of tumor growth and on the course of the hydrocephalic syndrome.

\section{CONCLUSIONS}

1. Hydrocephalus is detected in $62 \%$ of patients with malignant brain tumors of various localization, size and histostructure. In this case, the development of hydrocephalus leads to the appearance or acceleration of the course of the hypertensive syndrome and the aggravation of the patient's condition.

2. The incidence of hydrocephalus does not depend on the degree of tumor anaplasia, but depends on the child's age, location, size and type of tumor growth.

3. Occlusion of the cerebrospinal fluid is the main cause of hydrocephalus in patients with malignant OGM $-75 \%$, however, 1.6 times less frequently than in patients with benign OGM.

4. After removal of malignant brain tumors, regression of hydrocephalic syndrome is achieved in $67.2 \%$ of cases, depending on the completeness of tumor removal.

5. Preservation of dropsy of the brain after total or subtotal removal of the tumor is due to impaired resorption of CSF and in $10 \%$ of cases - hyperproduction of cerebrospinal fluid.

6. The use of ventriculo-peritoneal shunting made it possible to achieve control over hydrocephalus in $88.2 \%$ of cases. However, in view of the high incidence of postoperative complications, ventriculoperitoneal shunting should also have limited application. Simultaneous ventriculo-peritoneal shunting with removal of brain tumor is not advisable.

7. The use of shunts with an antimetastatic barrier containing an adsorbent filter can significantly reduce the risk of dissemination blastomatous cells with cerebrospinal fluid flow.

8. With the progression of hydrocephalus in the long-term period in patients after removal of a malignant brain tumor, it is necessary to exclude a relapse of the blastomatous process.

\section{REFERENCES}

1. Bersnev, V.P. Surgical treatment of tumors of the posterior cranial fossa in children / V.P. Bersnev, A.G. Zemskaya, Khachatryan V.A. // Problems of neurosurgery. - SPb., 2000 .- S. 166 - 168.

2. Gasparyan, S.S. On the mechanisms of hydrocephalus in patients with parasagittal meningiomas / S.S. Gasparyan, A.E. Razumovsky, A.R. Shakhnovich, G.A. Gabibov, V.N. Kornienko // Neurosurgical questions. - 1985. - No. 1. - P. 13 - 19.

3. Kim, Won Gi. Hydrocephalus in supratentorial brain tumors: Author's abstract. diss. ... Cand. honey. Sci. / Kim Won Gi. - SPb., 1996 .-- 25 p.

4. Kim, A.V. Diagnostics and treatment of hypertensive-hydrocephalic syndrome in children with tumors of the posterior cranial fossa: Author's abstract. diss. ... Ph.D. Sciences / Kim A.V. - SPb., 2004 -- 25 p.

5. Kommunarov, V.V. The choice of the parameters of the implantable drainage system in the treatment of hydrocephalus: Abstract of the thesis. diss. ... Cand. honey. Science / V.V. Kommunarov. - SPb., 2003 .-$25 \mathrm{p}$.

6. Ozerov, S.S. Medulloblastomas. Complex treatment problems / S.S. Ozerova, S.K. Gorelyshev, E.A. Khukhlaeva et al. // 
Bulletin of Ukrainian Asociatsii neurosurgery. - Kiev, 1998. - No. 5. - P. 158

7. Khachatryan, V.A. CSF shunt operations in the treatment of hydrocephalus of various etiology / V.A. Khachatryan // IV All-Union Congress of Neurosurgeons. - M., 1998 .-- S. $43-46$.

8. Khachatryan, V.A. Pathogenesis and surgical treatment of hypertensive hydrocephalus: Khachatryan, V.A. Hydrocephalus (pathogenesis, diagnosis, surgical treatment)/ V.A. Khachatryan, V.P. Bersnev, Sh.M. Safin, Yu.A. Orlov, T.N. Trofimova // SPb., 1999.

9. Albright $L$. The value of precraniotomy shunts on children with posterior fossa tumors // Clin. Neurosurg. - 1983. - V.20. P.278-285.

10. Atlas M.D. Evolution of the management of hydrocephalus associated with acoustic neuroma. Atlas MD, Perez de Tagle JR, Cook JA, Sheehy JP, Fagan PA. Laryngoscope. - 1996. - Vol. 106 (2 Pt 1). - P. 204-206.

11. Chapman P.H. Telemetric ICP monitoring after surgery for posterior fossa and third ventricular tumors / P.H. Chapman, E. Cosman, M. Arnold // J. Neurosurg. - 1984. Vol. 60, no. 6. -R. 649-651.

12. Chang C.H., Housepian E.M., Herbert C Jr. An operative staging system and a megavoltage radiotherapeutic technic for cerebellar medulloblastomas // Radiology. -1969. -Vol. 93. -P. 1351-1359.

13. Culley D.J. An analysis of factors determining the need for ventriculoperitoneal shunts after posterior fossa tumor surgery in children / D.J. Culley, M.S. Berger, D. Shaw // Neurosurg. -1994. Vol. 34. -P. 402 - 408.

14. Epstein F. Pediatric posterior fossa tumor: hazards of the "preoperative" shunt / F.
Epstein, R. Murali // Neurosurg. -1978. -Vol. 3. -P. 348-350.

15. Fiorillo A. Shunt-related abdominal metastases in an infant with medulloblastoma: long-term remission by systemic chemotherapy and surgery. Fiorillo A, Maggi G, Martone A, Migliorati R, D'Amore R, Alfieri E, Greco N, Cirillo S, Marano I. // J Neurooncol. - 2001. - Vol. 52 (3). - P. 273-276.

16. Griwan M.S. Value of precraniotomy shunts in children with posterior fossa tumours / M.S. Griwan, B.S. Sharma, R.K. Mahajan, V.K. Kak // Childs Nerv. Syst. - 1993. - Vol. 9 (8). - P. $462-465$.

17. Inamasu J., Nakamura Y., Saito R., Kuroshima Y., Mayanagi K., Orii M. and Ichikizaki K. Postoperative communicating hydrocephalus in patients with supratentorial malignant glioma. Clin Neurol Neurosurg 2003; 106: 9-15

18. Haas J. Ventricular shunts and drainage in the management of posterior fossa tumors. In posterior fossa tumors (ed. Raimondi A.J., Choux M., De Ricco E.), 1993, p. 80-85.

19. Khachatryan W.A. On the pathogenesis of hypertensive hydrocephalus // 62nd Ann. Meet. Am. Ass. Neurol. Surg., San Diego. 1994. - P.343-344.

20. Kumar V. Ventriculo-peritoneal shunt requirement in children with posterior fossa tumours: an 11-year audit / V. Kumar, K. Phipps, W. Harkness, R.D. Hayward // Br J. Neurosurg. - 1996. - Vol. 10 (5). - P. 467470.

21. Lee $M$. Management of hydrocephalus in children with medulloblastoma: prognostic factors for shunting / M. Lee, J.H. Wisoff, R. Abbott // Pediatr. Neurosurg. - 1994. - Vol. 20. - P. 240 - 247.

22. Raimondi A.J. Hydrocephalus and infratentorial tumors. Incidence, clinical 
pictures, and treatment / A.J. Raimondi, T.

Tomita // J. Neurosurg. - 1981. - Vol. 55 .-- P.

$174-182$.

23. Sainte-Rose Ch. Management of hydrocephalus in pediatric patients with posterior fossa tumors: the role of endoscopic third ventriculostomy / Ch. Sainte-Rose, G. Cinalli, F.E. Roux, W. Maixner, P.D. Chumas, M. Mansour, A. Carpentier, M. Bourgeois, M. Zerah, A. Pierre-Kahn, D. Renier // Neurosurg. Focus 1999. - Vol. 7 (4). - Art. 3.

24. Wellons J.C. Long-term control of hydrocephalus via endoscopic third ventriculostomy in children with tectal plate gliomas / J.C. Wellons, R.S. Tubbs, J.T. Banks, B. Grabb, J.P. Blount // Neurosurg. 2002.

Vol. 51 (1). - P. $63-67$.

25. Yamini B., Refai D., Rubin C. et al. Initial endoscopic management of pineal region tumors and associated hydrocephalus: clinical series and literature review. J.Neurosurgery 2004; 100(5 ): 437-41. 